\title{
ANYONS AND SINGULAR GAUGE TRANSFORMATIONS
}

\author{
A FOERSTER and H.O GIROTTI \\ Instituto de Fisica, Untversidade Federal do Rıo Grande do Sul, Calxa Postal 15051, 91500 Porto Alegre, RS, Brazll
}

Recelved 14 August 1989

\begin{abstract}
A $(2+1)$-dimensional theory of charged scalar particles coupled to an abelian gauge field with Chern-Simons term in the action is canonically quantized in the Coulomb and superaxial gauges The gauge transformation linkıng these two gauges is shown to be singular Then, the superaxial gauge excitations are found to obey fractional statistics We demonstrate that this effect does not arise when the conventional term $-\frac{1}{4} F^{\mu \nu} F_{\mu \nu}$ is present in the action
\end{abstract}

As is known gauge theories fall into the class of constrained systems, the first-class constraints acting as independent generators of gauge transformations [1] In order to quantize the theory one must first suppress all gauge freedom by bringing into the game subsidiary (gauge) conditions, which together with the constraints form a set of second-class constraints The classical-quantum transition is then performed by abstracting the equal-tıme commutators (ETC's) from the corresponding Dirac brackets (DB's), the constraints and gauge conditions thereby translating into strong operator relations [2,3] This is the so called Dirac bracket quantization procedure (DBQP)

We shall call $\Sigma$ and $\Sigma_{\mathrm{G}}$ the hypersurfaces defined in phase-space $(\Gamma)$ by the constraints and gauge cond1tions, respectively As is clear from above, the DBQP only makes sense in the physical space $\Gamma^{*} \equiv \Sigma \cap \Sigma_{\mathrm{G}}$ [2,3] The unconstrained canonical variables spanning $\Gamma^{*}$ will be designated by $\left\{q^{*}\right\}$ and $\left\{p^{*}\right\}$ Gauge invariance demands that a change of gauge, $\Sigma_{\mathrm{G}} \rightarrow \Sigma_{\mathrm{G}^{\prime}}$, should merely reduce to a canonical transformation in $\Gamma^{*}$ Hence, for all regular gauge transformations, $q^{*}$ and its canonical transform $\left(q^{* \prime}\right.$, say) will describe excitations obeying the same statistics

On the other hand, if the gauge transformation is singular $q^{*}$ and $q^{* \prime}$ may describe excitations obeying different statistics The main purpose of this paper is

\footnotetext{
* Supported in part by Conselho Nacional de Desenvolvimento Científico e Tecnológico (CNPq), Brazil
}

to show that such singular transformations in fact occurs in the $((2+1)$-dimensional) Chern-Simons theory whose lagrangian density reads [4]

$$
\mathscr{L}=\left(\overline{\mathrm{D}_{\mu} \phi}\right)\left(\mathrm{D}^{\mu} \phi\right)+\frac{\theta}{4 \pi^{2}} \epsilon_{\mu \nu \lambda} A^{\mu} \partial^{\nu} A^{\lambda},
$$

where the fully antisymmetric tensor $\epsilon^{\mu \nu \lambda} 1$ s normal1zed such that $\epsilon^{012}=1$, the metric is $g^{00}=-g^{11}=$ $-g^{22}=+1, \theta$ is a dimensionless parameter and $\bar{\phi}$ denotes the complex conjugate of $\phi$ By assumption, the scalar field $\phi$ is minımally coupled to the abelian gauge potentral $A^{\mu}, 1$ e., $\mathrm{D}^{\mu}=\partial^{\mu}-1 A^{\mu}$

We shall quantize this model in the Coulomb and superaxial $[5,6]$ gauges It will be shown, afterwards, that the gauge transformation linkıng these two gauges is singular Then, $\phi$ will be seen to verify a bosonic equal-tıme algebra in the Coulomb gauge and a graded ("anyonic" [7]) equal-tıme algebra in the superaxral gauge Furthermore, we will demonstrate that the absence of the term $-\frac{1}{4} F_{\mu \nu} F^{\mu \nu}\left(F^{\mu \nu}=\partial^{\mu} A^{\nu}-\partial^{\nu} A^{\mu}\right)$ in (1) is at the root of this statistical transmutation $[7,8]$

Withın the hamiltonian framework the system under analysis is fully characterized by the canonical hamıltonian

$H_{0}=\int \mathrm{d}^{2} z\left[p \bar{p}+\left(\overline{\mathrm{D}^{\prime} \phi}\right)\left(\mathrm{D}^{\prime} \phi\right)\right]$,

the primary first-class constraint

$P_{0} \equiv \pi_{0} \approx 0$, 
the primary second-class constraints $\left(\epsilon^{\mathrm{l} J}=\epsilon^{0 \mathrm{uJ}}\right)$

$P_{l} \equiv \pi_{\imath}-\frac{\theta}{4 \pi^{2}} \epsilon^{l \jmath} A^{j} \approx 0, \quad l=1,2$,

and the secondary first-class constraint

$S_{0} \equiv 1(\bar{\phi} \bar{p}-p \phi)+\partial^{i} \pi_{t}+\frac{\theta}{4 \pi^{2}} \epsilon^{l} \partial^{i} A^{J}$

Here, we have designated by $\pi_{\mu}, p$ and $\bar{p}$ the momenta canonically conjugate to $A^{\mu}, \phi$ and $\bar{\phi}$, respectively. Due to the presence of the Chern-Simons term neither $A^{t}$ nor $\pi_{l}$ are gauge invariant quantities As usual, the sector of phase space spanned by $A^{0}, \pi_{0}$ can be elıminated from $\Gamma, \pi_{0}$ is fixed by the constraint condition (3) while $A^{0}$ acts as the Lagrange multiplier of $S_{0}$ and will be determined, after gauge fixing, as a function of the remaining canonical variables.

The quantization of the system in the Coulomb gauge, $\chi \equiv \partial^{t} A^{t} \approx 0$, is straightforward The set of constraints $\psi_{l} \equiv P_{l} \approx 0, l=1,2, \psi_{3} \equiv S_{0} \approx 0, \psi_{4} \equiv \chi \approx 0$ is second-class and one can, therefore, introduce the Dirac bracket in the standard manner Then, through the DBQP one arrives to the following set of ETC's $\# 1$

$$
\begin{aligned}
& {[\phi(x), p(y)]=1 \hbar \delta(x-y),} \\
& {[\phi(x), \phi(y)]=\left[\phi(x), \phi^{\dagger}(y)\right]=\left[\phi(x), p^{\dagger}(y)\right]} \\
& \quad=[p(x), p(y)]=\left[p(x), p^{\dagger}(y)\right]=0, \\
& {\left[\phi(x), A^{l}(y)\right]=\frac{2 \pi^{2}}{\theta} \hbar \phi(x) \epsilon^{\jmath j} \frac{\partial}{\nabla_{x}^{2}} \delta(x-y),} \\
& {\left[\phi(x), \pi_{l}(y)\right]=-\frac{\hbar}{2} \phi(x) \frac{\partial_{l}^{x}}{\nabla_{x}^{2}} \delta(x-y),} \\
& {\left[p(x), A^{i}(y)\right]=-\frac{2 \pi^{2}}{\theta} \hbar p(x) \epsilon^{y} \frac{\partial_{J}^{x}}{\nabla_{x}^{2}} \delta(x-y),} \\
& {\left[p(x), \pi_{l}(y)\right]=\frac{\hbar}{2} p(x) \frac{\partial_{l}^{x}}{\nabla_{x}^{2}} \delta(x-y),} \\
& {\left[A^{\prime}(x), \pi_{j}(y)\right]=0,} \\
& {\left[\pi_{t}(x), \pi_{j}(y)\right]=0}
\end{aligned}
$$

This algebra carries, as it must, the strong operator relations $\psi_{a}=0, a=1,2,3,4$

The unconstrained phase space variables spanning $\Gamma^{*}$ can be easily found Indeed, the second class con-

\#1 To simplify the notation we shall not distınguish between a quantum field operator and its classical counterpart straints $P_{l}=0, l=1,2$ (see eq (4)) allow for the elimination of $\pi_{1}$ and $\pi_{2}$ in favor of $A^{2}$ and $A^{1}$, respectively, while the Coulomb condition $\partial^{l} A^{\prime} \approx 0$ enables one to cast $A^{l}$ as a purely transversal field This together with eq $(5)$ lead to

$A^{l}(\boldsymbol{x})=-\frac{2 \pi^{2}}{\theta} \epsilon^{l j} \frac{\partial_{J}^{x}}{\nabla_{x}^{2}} J^{0}(\boldsymbol{x})$,

where

$J^{0}(\boldsymbol{x}) \equiv 1\left(\phi^{\dagger} p^{\dagger}-p \phi\right)$

One then concludes that the fields $A^{t}, \pi_{l}, l=1,2$, can be completely eliminated in favor of $\phi, \phi^{\dagger}, p$ and $p^{\dagger}$, which, according to $(6 \mathrm{a}),(6 \mathrm{~b})$, constitute a canonlcal basis in $\Gamma^{*}$ describing bosonic excitations

All Poincaré generators can of course be written solely in terms of the independent fields In particular, the hamiltonian $(H)$, the linear momentum $\left(P^{k}\right)$ and the angular momentum $(J)$ operators are given by

$$
\begin{aligned}
H & \equiv \int \mathrm{d}^{2} x \theta^{00}(\boldsymbol{x}) \\
& =\int \mathrm{d}^{2} x\left\{p(\boldsymbol{x}) p^{\dagger}(\boldsymbol{x})+\left[\partial_{\iota} \phi^{\dagger}(\boldsymbol{x})\right][\partial \phi(\boldsymbol{x})]\right\} \\
& +\frac{\pi^{2}}{\theta^{2}} \int \mathrm{d}^{2} x \mathrm{~d}^{2} u \mathrm{~d}^{2} v \frac{1}{|\boldsymbol{x}-\boldsymbol{u}|^{2}} \phi^{\dagger}(\boldsymbol{x}) j^{0}(\boldsymbol{u}) j^{0}(\boldsymbol{v}) \phi(\boldsymbol{x}) \\
& -\frac{1 \pi}{\theta} \epsilon^{\jmath l} \int \mathrm{d}^{2} x \mathrm{~d}^{2} u \frac{\left(x^{l}-u^{l}\right)}{|\boldsymbol{x}-\boldsymbol{u}|^{2}} \\
& \times\left\{\left[\partial_{\jmath} \phi(\boldsymbol{x})\right] J^{0}(\boldsymbol{u}) \phi(\boldsymbol{x})-\phi^{\dagger}(\boldsymbol{x}) J^{0}(\boldsymbol{u})\left[\partial_{\jmath} \phi(\boldsymbol{x})\right]\right\},
\end{aligned}
$$

$$
\begin{aligned}
P^{k} & \equiv \int \mathrm{d}^{2} x \theta^{0 k}(\boldsymbol{x})=\int \mathrm{d}^{2} x\left[\left(\partial^{k} \phi^{\dagger}\right) p^{\dagger}+p\left(\partial^{k} \phi\right)\right] \\
& -\frac{\pi}{\theta} \epsilon^{k j} \int \mathrm{d}^{2} x \mathrm{~d}^{2} u \frac{\left(x^{\jmath}-u^{\jmath}\right)}{|\boldsymbol{x}-\boldsymbol{u}|^{2}} J^{0}(\boldsymbol{x}) J^{0}(\boldsymbol{u}) \\
& +\frac{\hbar \pi}{\theta} \epsilon^{k J} \int \mathrm{d}^{2} x \mathrm{~d}^{2} u \frac{\left(x^{\prime}-u^{\prime}\right)}{|\boldsymbol{x}-\boldsymbol{u}|^{2}} J^{0}(\boldsymbol{x}) \delta(\boldsymbol{x}-\boldsymbol{u}),
\end{aligned}
$$

$$
\begin{aligned}
J & \equiv \int \mathrm{d}^{2} x \epsilon^{j k} x^{j} \theta^{0 k}(x) \\
& =\int \mathrm{d}^{2} x \epsilon^{j k} x^{\jmath}\left[\left(\partial^{k} \phi^{\dagger}\right) p^{\dagger}+p\left(\partial^{k} \phi\right)\right] \\
& +\frac{\pi}{2 \theta} Q^{2}-\frac{\hbar \pi}{2 \theta} Q,
\end{aligned}
$$


where $Q \equiv \int \mathrm{d}^{2} x J^{0}$ and we have used $\left(\nabla_{x}^{2}\right)^{-1} \delta(x-y)$ $=(4 \pi)^{-1} \ln \left(|x-y|^{2}\right)+$ constant The last two terms on the right-hand side of (10) can be seen to vanish, however, their corresponding integrands make a meanıngful contribution to $\theta^{0 k}$. In spite of ordering problems, we have been able of constructıng a set of hermitean Poincaré density operators $\theta^{\mu \nu}$ obeying, as they must, the Dirac-Schwinger equation

$$
\begin{aligned}
& {\left[\theta^{00}(\boldsymbol{x}), \theta^{00}(\boldsymbol{y})\right]} \\
& \quad=-1 \hbar\left[\theta^{0 k}(\boldsymbol{x})+\theta^{0 k}(\boldsymbol{y})\right] \partial_{k}^{x} \delta(\boldsymbol{x}-\boldsymbol{y})
\end{aligned}
$$

The verification of this last equation is straightforward but tedious.

As first noticed by Hagen [9], the operator $\phi$ developes a rotational anomaly already at the classical level,

$$
\begin{aligned}
\bar{\delta} \phi(\boldsymbol{x}) & \equiv-\frac{1}{\hbar} \delta \omega[\phi(\boldsymbol{x}), J] \\
& =-\delta \omega\left[\epsilon^{j k} x^{j} \partial_{k} \phi+(1 \pi / \theta) Q \phi\right],
\end{aligned}
$$

where the infinitesimal rotation is parametrized by $\delta \omega$ Nevertheless, the presence of this anomaly does not alter the fact that the charged field $\phi$ obeys Bose statistics [9] This is how the situation looks like in the Coulomb gauge

We now turn into quantızing the theory in the fully fixed axial gauge (the superaxial gauge) specified by the subsidiary conditions [5]

$A_{\mathrm{s}}^{2}\left(x^{0}, x^{1}, x^{2}\right)=0$,

$A_{\mathrm{s}}^{1}\left(x^{0}, x^{1}, x_{(0)}^{2}\right)=0$,

$A_{\mathrm{s}}^{0}\left(x^{0}, x_{(0)}^{1}, x_{(0)}^{2}\right)=A^{0}\left(x^{0}, x_{(0)}^{1}, x_{(0)}^{2}\right)$

Here, $x_{(0)} \equiv\left(x_{(0)}^{1}, x_{(0)}^{2}\right)$ denotes some arbitrary fixed point The subscript " $s$ " Identifies the field variables belonging to the superaxial gauge; Coulomb gauge field variables remain without gauge identification The superaxial gauge can be reached from the Coulomb gauge via the following operator gauge transformation [5]

$$
\begin{aligned}
& A_{\mathrm{s}}^{\mu}(x)=A^{\mu}(x)+\partial^{\mu} A(x), \\
& \phi_{\mathrm{s}}(x)=\exp [1 \Lambda(x)] \phi(x), \\
& p_{\mathrm{s}}(x)=p(x) \exp [-1 A(x)], \\
& \phi_{\mathrm{s}}^{\dagger}(x)=\phi^{\dagger}(x) \exp [-1 \Lambda(x)],
\end{aligned}
$$

$$
p_{\mathrm{s}}^{\dagger}(x)=\exp [\mathbf{1} \Lambda(x)] p^{\dagger}(x),
$$

where

$$
\begin{gathered}
A(x)=\int_{x_{(0)}^{2}}^{x^{2}} \mathrm{~d} x^{\prime 2} A^{2}\left(x^{0}, x^{1}, x^{\prime 2}\right) \\
+\int_{x_{(0)}^{1}}^{x^{1}} \mathrm{~d} x^{\prime 1} A^{1}\left(x^{0}, x^{\prime 1}, x_{(0)}^{2}\right)
\end{gathered}
$$

The order of factors in (14) is fixed. We emphasize that $A$ does not commute with the charged fields and that this is due to the fact that $A^{\prime}$ does not commute with $\phi$ and $p$ (see eqs (6c) and (6e)) A straightforward calculation shows that

$$
\begin{aligned}
& {[\Lambda(x), \phi(y)]=-\frac{\pi \hbar}{\theta} F(x, y) \phi(y),} \\
& {[A(x), p(y)]=\frac{\pi \hbar}{\theta} F(x, y) p(y),}
\end{aligned}
$$

where

$$
\begin{aligned}
& F(\boldsymbol{x}, \boldsymbol{y})=\epsilon\left(x^{1}-y^{1}\right) \\
& \times\left[\arctan \left(\frac{x^{2}-y^{2}}{\left|x^{1}-y^{1}\right|}\right)-\arctan \left(\frac{x_{(0)}^{2}-y^{2}}{\left|x^{1}-y^{1}\right|}\right)\right] \\
& -\epsilon\left(x_{(0)}^{2}-y^{2}\right) \\
& \quad \times\left[\arctan \left(\frac{x^{1}-y^{1}}{\left|x_{(0)}^{2}-y^{2}\right|}\right)-\arctan \left(\frac{x_{(0)}^{1}-y^{1}}{\left|x_{(0)}^{2}-y^{2}\right|}\right)\right]
\end{aligned}
$$

and $\epsilon(x)$ is the sign function

The fields $\phi_{\mathrm{s}}, \phi_{\mathrm{s}}^{\dagger}, p_{\mathrm{s}}$ and $p_{\mathrm{s}}^{\dagger}$ are the unconstrained variables spanning the physical space in the superaxial gauge formulation of the theory In fact, as it happens in the case of the Coulomb gauge, $A_{\mathrm{s}}^{t}, \pi_{t s}$, $\imath=1,2$, can be elimınated in terms of $\phi_{\mathrm{s}}, \phi_{\mathrm{s}}^{\dagger}, p_{\mathrm{s}}$ and $p_{\mathrm{s}}^{\dagger}$ This come about because both terms on the righthand side of (14a) only involve the Coulomb fields $A^{t}, \imath=1,2$, whose elımınation in terms of the gauge invariant operator $J^{0}$ was already carried out in (8) As follows from eqs (14), (15) and (6), the ETC's in the superaxial gauge can be found from the corresponding ones in the Coulomb gauge For the ETC's involving only the independent variables one obtains 


$$
\begin{aligned}
& {\left[\phi_{\mathrm{s}}(\boldsymbol{x}), \phi_{\mathrm{s}}(\boldsymbol{y})\right]} \\
& \quad=\{\exp [(1 / \theta) \Delta(\boldsymbol{x}, \boldsymbol{y})]-1\} \phi_{\mathrm{s}}(\boldsymbol{y}) \phi_{\mathrm{s}}(\boldsymbol{x}), \\
& {\left[\phi_{\mathrm{s}}(\boldsymbol{x}), \phi_{\mathrm{s}}^{\dagger}(\boldsymbol{y})\right]} \\
& \quad=\{\exp [-(1 / \theta) \Delta(\boldsymbol{x}, \boldsymbol{y})]-1\} \phi_{\mathrm{s}}^{\dagger}(\boldsymbol{y}) \phi_{\mathrm{s}}(\boldsymbol{x}), \\
& {\left[\phi_{\mathrm{s}}(\boldsymbol{x}), p_{\mathrm{s}}(\boldsymbol{y})\right]=1 \hbar \delta(\boldsymbol{x}-\boldsymbol{y})} \\
& \quad+\{\exp [-(1 / \theta) \Delta(\boldsymbol{x}, \boldsymbol{y})]-1\} p_{\mathrm{s}}(\boldsymbol{y}) \phi_{\mathrm{s}}(\boldsymbol{x}), \\
& {\left[\phi_{\mathrm{s}}(\boldsymbol{x}), p_{\mathrm{s}}^{\dagger}(\boldsymbol{y})\right]} \\
& \quad=\{\exp [(1 / \theta) \Delta(\boldsymbol{x}, \boldsymbol{y})]-1\} p_{\mathrm{s}}^{\dagger}(\boldsymbol{y}) \phi_{\mathrm{s}}(\boldsymbol{x}), \\
& {\left[p_{\mathrm{s}}(\boldsymbol{x}), p_{\mathrm{s}}(\boldsymbol{y})\right]} \\
& \quad=\{\exp [(1 / \theta) \Delta(\boldsymbol{x}, \boldsymbol{y})]-1\} p_{\mathrm{s}}(\boldsymbol{y}) p_{\mathrm{s}}(\boldsymbol{x}), \\
& {\left[p_{\mathrm{s}}(\boldsymbol{x}), p_{\mathrm{s}}^{\dagger}(\boldsymbol{y})\right]} \\
& \quad=\{\exp [-(1 / \theta) \Delta(\boldsymbol{x}, \boldsymbol{y})]-1\} p_{\mathrm{s}}^{\dagger}(\boldsymbol{y}) p_{\mathrm{s}}(\boldsymbol{x}),
\end{aligned}
$$

where

$$
\Delta(\boldsymbol{x}, \boldsymbol{y})=-\hbar \pi[F(\boldsymbol{x}, \boldsymbol{y})-F(\boldsymbol{y}, \boldsymbol{x})]
$$

We mention that the equal-tıme superaxial algebra also carries the constraints (4), (5) and the gauge conditions [13] as strong operator relations

From eqs (6a), (6b) and (18) follow that the structure of the ETC's involving only unconstrained variables is not preserved under the transformation (14) Hence, this transformation is singular (noncanonical) Moreover, the ETC's (18) can be easily casted in the form of graded equal-time commutation relations [4],

$$
\begin{aligned}
& \phi_{\mathrm{s}}(\boldsymbol{x}) \phi_{\mathrm{s}}(\boldsymbol{y})-\exp [(1 / \theta) \Delta(\boldsymbol{x}, \boldsymbol{y})] \phi_{\mathrm{s}}(\boldsymbol{y}) \phi_{\mathrm{s}}(\mathrm{s}) \\
& \quad=0 \\
& \quad \phi_{\mathrm{s}}(\boldsymbol{x}) \phi_{\mathrm{s}}^{\dagger}(\boldsymbol{y})-\exp [-(1 / \theta) \Delta(\boldsymbol{x}, \boldsymbol{y})] \phi_{\mathrm{s}}^{\dagger}(\boldsymbol{y}) \phi_{\mathrm{s}}(\boldsymbol{x}) \\
& \quad=0 \\
& \phi_{\mathrm{s}}(\boldsymbol{x}) p_{\mathrm{s}}(\boldsymbol{y})-\exp [-(1 / \theta) \Delta(\boldsymbol{x}, \boldsymbol{y})] p_{\mathrm{s}}(\boldsymbol{y}) \phi_{\mathrm{s}}(\boldsymbol{x}) \\
& \quad=1 \hbar \delta(\boldsymbol{x}-\boldsymbol{y}) \\
& \phi_{\mathrm{s}}(\boldsymbol{x}) p_{\mathrm{s}}^{\dagger}(\boldsymbol{y})-\exp [(1 / \theta) \Delta(\boldsymbol{x}, \boldsymbol{y})] p_{\mathrm{s}}^{\dagger}(\boldsymbol{y}) \phi_{\mathrm{s}}(\boldsymbol{x}) \\
& \quad=0
\end{aligned}
$$

The excitations described by the field $\phi_{\mathrm{s}}$ obey, then, fractional statistics We strongly emphasize that the lack of commutativity of $A^{J}$ with $\phi$ and $p_{1}$ at the root of this effect (see eqs (16), (19) and (20)) For arbitrary $\boldsymbol{x}_{(0)}, \Delta(\boldsymbol{x}, \boldsymbol{y})$ is a complicated function of $\boldsymbol{x}$ and $\boldsymbol{y}$, as seen from (17) and (19) Nevertheless, $\Delta(\boldsymbol{x}$, $y$ ) acquires a simple form for some particular superaxial gauges For instance, the election $x_{(0)}^{2}=+\infty$ leads to $(-\hbar \pi)^{-1} \Delta(x, y)=\epsilon\left(x^{1}-y^{1}\right)(\pi+2 k \pi)$, where $k$ is an integer Notice that the singular gauge transformation (14) does not remove the interaction Indeed, in terms of the superaxial gauge var1ables, the hamıltonian reads

$$
\begin{aligned}
H & =\int \mathrm{d}^{2} x\left\{p_{\mathrm{s}}(\boldsymbol{x}) p_{\mathrm{s}}^{\dagger}(\boldsymbol{x})+\left[\partial_{l} \phi_{\mathrm{s}}^{\dagger}(\boldsymbol{x})\right]\left[\partial_{l} \phi_{\mathrm{s}}(\boldsymbol{x})\right]\right\} \\
& -\frac{4 \pi^{4}}{\theta^{2}} \int \mathrm{d}^{2} x \int_{x_{(0)}^{2}}^{x^{2}} \mathrm{~d} u \int_{x_{(0)}^{2}}^{x^{2}} \mathrm{~d} v \phi_{\mathrm{s}}^{\dagger}(\boldsymbol{x}) \\
& \times J^{0}\left(x^{1}, u\right) J^{0}\left(x^{1}, v\right) \phi_{\mathrm{s}}(\boldsymbol{x}) \\
& -1 \frac{2 \pi^{2}}{\theta} \int \mathrm{d}^{2} x \int_{x_{(0)}^{2}}^{x^{2}} \mathrm{~d} u\left\{\left[\partial_{1} \phi_{\mathrm{s}}^{\dagger}(\boldsymbol{x})\right] J^{0}\left(x^{1}, u\right) \phi_{\mathrm{s}}(\boldsymbol{x})\right. \\
& \left.-\phi_{\mathrm{s}}^{\dagger}(\boldsymbol{x}) j^{0}\left(x^{1}, u\right)\left[\partial_{1} \phi_{\mathrm{s}}(\boldsymbol{x})\right]\right\}
\end{aligned}
$$

We study at last the modifications induced by the term $-\frac{1}{4} F^{\mu \nu} F_{\mu \nu}$ when added to the lagrangian (1) First to all the introduction of a dimensional coupling constant $e$ is now necessary This changes the covariant derivative into $\mathrm{D}^{\mu}=\partial^{\mu}-1 e A^{\mu}$ Furthermore, $\theta$ also becomes a dimensional parameter Within the hamiltonian formalısm the new system is completely characterized by the canonical hamiltonian \#2

$$
\begin{aligned}
& H_{0}^{\prime}=\int \mathrm{d}^{2} z\left[\frac{1}{2} \pi_{\imath} \pi_{t}-\left(\theta / 4 \pi^{2}\right) \pi_{t} \epsilon^{i k} A^{k}+\frac{1}{4} F^{\prime \jmath} F^{\prime \jmath}\right. \\
& \left.\quad+\left(\theta^{2} / 32 \pi^{4}\right) A^{J} A^{j}+p \bar{p}+\left(\overline{\mathrm{D}^{\prime} \phi}\right)\left(\mathrm{D}^{\prime} \phi\right)\right],
\end{aligned}
$$

the primary first-class constraint

$P_{0}^{\prime} \equiv \pi_{0} \approx 0$,

and the secondary first-class constraint

$S_{0}^{\prime} \equiv 1 e(\bar{\phi} \bar{p}-p \phi)+\partial^{i} \pi,+\frac{\theta}{4 \pi^{2}} \epsilon^{\prime j} \partial^{\prime} A^{\prime}$

The second-class constraints (4) are no longer present and, as consequence, the direct connection

\#2 A similar model, but with fermions instead of scalar fields, was presented in ref $[10]$ 
between $\pi$ 's and $A$ 's, established by these constraints, disappears This is the main effect provoked by the addition of the term $-\frac{1}{4} F^{\mu \nu} F_{\mu \nu}$ The theory is then quantized in the Coulomb gauge As far as the ETC's are concerned, the essential difference with the previous case is that $(6 \mathrm{c}),(6 \mathrm{e}),(6 \mathrm{~g}),(6 \mathrm{~h})$ are, respectively, replaced by

$$
\begin{aligned}
& {\left[\phi(x), A^{l}(y)\right]=0} \\
& {\left[p(x), A^{l}(y)\right]=0} \\
& {\left[A^{l}(x), \pi_{j}(y)\right]=1 \hbar\left(\delta_{j}^{l}-\frac{\partial_{x}^{l} \partial_{x}^{\prime}}{\nabla_{x}^{2}}\right) \delta(x-y),} \\
& {\left[\pi_{l}(x), \pi_{j}(y)\right]} \\
& \quad=\frac{1 \hbar \theta}{4 \pi^{2}}\left(\frac{\epsilon^{k l} \partial_{x}^{k} \partial_{x}^{\prime}-\epsilon^{k j} \partial_{x}^{k} \partial_{x}^{l}}{\nabla_{x}^{2}}\right) \delta(x-y)
\end{aligned}
$$

Hence, the operator $A(x)$, performing the transition from the Coulomb to the superaxial gauge, now commutes with $\phi(x), \phi^{\dagger}(x), p(x)$ and $p^{\dagger}(x)$ implying that the c-number function $F(x, y)=0$ (see eqs (16)) Then, the conclusion is that when $-\frac{1}{4} F^{\mu \nu} F_{\mu \nu}$ 1s present $\phi_{\mathrm{s}}$ and $\phi$ both describe bosonic excitations (see eqs. (18), (19) and (20))

We are indebted to Professor A.J da Silva for many illuminating discussions

\section{References}

[1] PA M Dirac, Lectures on quantum mechanics, Belfer Graduate School of Science, Yeshiva University (New York, 1964)

[2] E S Fradkın and G A Vllkovısky, CERN preprint TH2332 (1977), unpublished

[3] HO Grott1, Classical and quantum dynamics of constrained systems, lectures, in Proc Vth Jorge Andre Swleca Summer School (São Paulo, Brazl, 1989), to appear

[4] G W Semenoff, Phys Rev Lett 61 (1988) 517

[5] H O Girott1 and H J Rothe, Phys Lett B 115 (1982) 257

[6] T J M Simões and HO Girottı, Ann Phys (NY) 169 (1986) 1

[7] R Mackenzie and F Wilczek, Intern J Mod Phys A 3 (1988) 2827

[8] A Polyakov, Mod Phys Lett A 3 (1988) 325

[9] C R Hagen, Phys Rev D 31 (1985) 2135

[10] S Deser, R Jackıw and S Templeton, Ann Phys (NY) 140 (1982) 372 